\title{
Polymerized LB Films Imaged with a Combined Atomic Force Microscope-Fluorescence Microscope
}

\author{
Constant A. J. Putman, ${ }^{*}$ Helen G. Hansma, Hermann E. Gaub, ${ }^{\dagger}$ and \\ Paul K. Hansma
}

\author{
Department of Physics, University of California, Santa Barbara, California 93106
}

Received March 16, 1992. In Final Form: June 23, 1992

\begin{abstract}
The first results obtained with a new stand-alone atomic force microscope (AFM) integrated with a standard Zeiss optical fluorescence microscope are presented. The optical microscope allows location and selection of objects to be imaged with the high-resolution AFM. Furthermore, the combined microscope enables a direct comparison between features observed in the fluorescence microscope and those observed in the images obtained with the AFM, in air or under liquid. The cracks in polymerized LangmuirBlodgett films of 10,12-pentacosadiynoic acid as observed in the fluorescence microscope run parallel to one of the lattice directions of the crystal as revealed by molecular resolution images obtained with the AFM. The orientation of these cracks also coincides with the polarization direction of the fluorescent light, indicating that the cracks run along the polymer backbone. Ripple-like corrugations on a submicrometer scale have been observed, which may be due to mechanical stress created during the polymerization process.
\end{abstract}

\section{Introduction}

Monolayer films of amphiphilic molecules can be created at an air-water interface. They can be transferred to atomically flat surfaces, such as mica and silicon, to form Langmuir-Blodgett (LB) films of stable quasicrystalline mono- or multilayers. Applications of the LB films are expected in nonlinear optics (second- and third-harmonic generation), biosensors, lubrication of magnetic tape, fabrication of integrated circuits using microlithography, and semiconductor devices such as photovoltaic cells. ${ }^{1-3}$

LB films are interesting not only in the field of material science, but also in biology and biochemistry. There the LB films are studied as model systems for biological membranes, which for the greater part consist of amphiphilic lipids. Most LB films are very fragile, and the lateral mobility of the components may be high. The mechanical stability of films of some new amphiphilic organic materials, which form LB films the way fatty acids, can be increased by chemically cross-linking the molecules. The molecules can be polymerized by irradiation with cobalt-60 $\gamma$ rays, electrons, or ultraviolet light. The polymer backbone features unique optical properties, which have been investigated by spectroscopical techniques. 4,5

A wide variety of other techniques has been used to study the structure and properties of LB films. Among them are X-ray ${ }^{6}$ and electron diffraction ${ }^{7-9}$ and recently

* To whom correspondence should be addressed at the Department of Applied Physics, University of Twente, P.O. Box 217, 7500 AE Enschede, The Netherlands. Phone/FAX: +31-53-893157/+31-53309549 .

+ Present address: Chemistry Department, Stanford University, Stanford, CA 94306.

(1) Agarwhal, V. K. Phys. Today 1988, June, 40.

(2) Roberts, G. G. In Electronics and Photonic Applications of Polymers; Bowden, M. J., Turner, S. R., Eds.; American Chemical Society: Washington, DC, 1988; pp 225-270.

(3) Swalen, J. D.; Allara, D. L.; Andrade, J. D.; Chandross, E. A.; Garoff, S.; Israelachvili, J.; McCarthy, T. J.; Murray, R.; Pease, R. F.; Rabolt, J. F.; Wynne, K. J.; Yu, H. Langmuir 1987, 3, 932.

(4) Warta, R.; Sixl, H. J. Chem. Phys. 1988, 88, 95.

(5) Rothberg, L.; Higashi, G. S.; Allara, D. L.; Garoff, S. Chem. Phys. Lett. 1987, 133, 67 .

(6) Kjaer, K.; Als-Nielsen, J.; Helm, C.; Tippman-Krayer, P.; Möhwald, H. J. Phys. Chem. 1989, 93, 3200 .

(7) Fischer, A.; Sackmann, E. Nature 1985, 313, 299.

(8) Lieser, G.; Tieke, B.; Wegnar, G. Thin Solid Films 1980, 68, 77. the scanning tunneling microscope (STM) ${ }^{10-13}$ and the atomic force microscope (AFM). ${ }^{14-20}$ The STM and AFM are the only techniques which give direct information on the nanometer-scale structure of the LB films, e.g., nanometer-scale defects ${ }^{17,19}$ or domain boundaries (Garnaes et al., private communication). The other techniques mentioned above give information on film structure averaged over areas ranging from square micrometers up to square millimeters. Little is known yet on the arrangement of the molecules in the crystals in correlation with the cracks in polymerized films observed in the fluorescence microscope. It has been suggested that the cracks are parallel to one crystal axis of the LB film. ${ }^{15}$

To stimulate the use of a new research tool, in this case the AFM, the new tool should, if possible, be integrated with a well-established tool in one apparatus. The optical microscope is the most widely used research tool for structural studies, especially in biology. An instrument combining an AFM and an optical microscope not only gives the high-resolution images of the AFM, but can also benefit from the expertise and knowledge in the field of optical microscopy, well established over the last few hundred years. The optical microscope not only helps in the location and selection of objects, but it also enables

(9) Garoff, S.; Deckman, H. W.; Dunsmuir, J. H.; Alvarez, M. S.; Bloch, J. M. J. Phys. (Paris) 1986, 47, 701.

(10) Coombs, J. H.; Pethica, J. B.; Welland, M. E. Thin Solid Films 1988, 159, 293.

(11) Braun, H. G.; Fuchs, H.; Schrepp, W. Thin Solid Films 1988, 159 , 301 .

(12) Hörber, J. K. H., Lang, C. A.; Hänsch, T. W.; Heckl, W. M.; Möhwald, H. Chem. Phys. Lett, 1988, 145, 151.

(13) Zasadzinski, J. A. N.; Hansma, P. K. Ann. N.Y. Acad. Sci. 1990 40,5363 . 930 .

(15) Weisenhorn, A. L.; Gaub, H. E.; Hansma, H. G.; Sinsheimer, R. L.; Kelderman, G. L.; Hansma, P. K. Scanning Microscopy 1990, 4, 511. (16) Hansma, H. G.; Weisenhorn, A. L.; Edmundson, A. B.; Gaub, H. E.; Hansma, P. K. Clin. Chem. (Winston-Salem, N.C.) 1991, 37, 1497. (17) Weisenhorn, A. L.; Egger, M.; Ohnesorge, F.; Gould, S. A. C.; Heyn, S.-P.; Hansma, H. G.; Sinsheimer, R. L.; Gaub, H. E.; Hansma, P. K. Langmuir 1991, 7, 8 .

(18) Hansma, H. G.; Gould, S. A. C.; Hansma, P. K.; Gaub, H. E.; Longo, M. L.; Zasadzinski, J. A. N. Langmuir 1991, 7, 1051.

(19) Meyer, E.; Howald, L.; Overney, R. M.; Heinzelmann, H.; Frommer, J.; Guntherodt, H.-J.; Wagner, T.; Schier, H.; Roth, S. Nature 1991, 349, 398.

(20) Goettgens, B. M.; Tillmann, R. W.; Radmacher, M.; Gaub, H. E. Langmuir 1992, 8, 1768 . 
a direct comparison between features observed in AFM images and those observed in the optical microscope.

In this study, we present the first results obtained with a new stand-alone AFM integrated with a standard Zeiss fluorescence microscope. A correlation can be made between the direction of the cracks in the LB films of 10,12-pentacosadiynoic acid as observed by the fluorescence microscope and the packing of the molecules in the crystal as revealed by the AFM.

\section{Materials and Methods}

LB Films. Monolayers of the polymerizable fatty acid 10,12-pentacosadiynoic acid (PCA) (ABCR, Karlsruhe, FRG) were formed at the surface of a home-built Langmuir trough and transferred by the standard Langmuir-Blodgett technique. ${ }^{1}$ The monolayer at the air-water interface (water as subphase, unless otherwise noted) was compressed to about $25 \mathrm{mN} / \mathrm{m}$ and polymerized by high-intensity UV irradiation ( $\mathrm{Hg}$ pen ray) for $30 \mathrm{~s}$. Two different types of samples were made. For the first type of samples, monolayers of PCA were transferred onto freshly cleaved muscovite mica (Mica New York Corp., New York) by vertical dipping. The hydrophilic headgroups adhere to the mica, and the hydrophobic tailgroups are exposed to air. This sample is imaged with the AFM in air. For the second type, bilayers of PCA were formed by vertical dipping. The bottom monolayer of Cd-PCA (subphase, $0.5 \mathrm{mM} \mathrm{CdCl}$ ) was UV-polymerized after transfer to mica. The top monolayer of PCA was transferred after UV polymerization. These samples were stored under water and imaged in the AFM under water, without having ever been out of the water.

Combined Atomic Force Microscope-Fluorescence Microscope. In Figure 1 a schematic diagram of the AFM integrated with the fluorescence microscope is shown. In contrast to the standard configuration of an AFM in which the cantilever with integrated tip $\mathrm{p}^{21}$ is stationary and the sample is scanned, the reverse is the case in this microscope. Other AFM-fluorescence microscope combinations which have been reported before also scan the sample. ${ }^{22,23} \mathrm{~A}$ bent wire holds down the cantilever on the cantilever holder attached to the piezo tube (an $x y z$-translator). The cantilever is placed at an angle of $10^{\circ}$ with the sample. The sample carrier (e.g., mica or glass) rests on the base with the sample facing down, and the tip is scanned over the sample on the bottom side of the sample carrier. At the rim of the piezo tube, between the tube and the Plexiglas cone, a flexible O-ring (not shown here) is positioned. This prevents liquid from leaking to the piezo and causing electrical short circuits between the electrodes. This $\mathrm{O}$-ring restricts the movement of the piezo tube by less than $10 \%$.

The deflection of the cantilever is detected with a focused laser beam from a laser diode reflecting off the backside of the cantilever toward a two-segment photodiode. ${ }^{24-28}$ A deflection of the cantilever gives a displacement (in Figure 1a, in and out of the plane defined by the paper) of the laser beam at the photodiode which, in first approximation, is proportional to the surface corrugation of the sample. A feedback loop keeps the deflection constant (apart from small variations not compensated by the feedback loop ${ }^{27}$ and therefore the applied force constant, by moving the cantilever up and down by applying a varying voltage to the $z$-electrode of the piezo tube, while raster scanning the cantilever. Displaying the applied voltages in a twodimensional matrix gives a topographic representation of the samples.

(21) Albrecht, T. R.; Akamine, S.; Carver, T. E.; Quate, C. F. J. Vac. Sci. Technol., A 1990, 8, 3386.

(22) Putman, C. A. J.; Van der Werf, K. O.; De Grooth, B. G.; Van Hulst, N. F.; Segerink, F. B.; Greve, J. Rev. Sci. Instrum. 1992, 63, 1914

(23) Radmacher, M.; Eberle, K.; Gaub, H. E. Ultramicroscopy 1992 42-44, 968

(24) Meyer, G.; Amer, N. M. Appl. Phys. Lett. 1988, 53, 2400.

(25) Alexander, S.; Hellemans, L.; Marti, O.; Schneir, J.; Elings, V. B.

Hansma, P. K.; Longmire, M. Gurley, J. J. Appl. Phys, 1989, 65, 164

(26) Putman, C. A. J.; De Grooth, B. G.; Van Hulst, N. F.; Greve, J. J. Appl. Phys. 1992, 72, 6.

(27) Putman, C. A. J.; Van der Werf, K. O.; De Grooth, B. G.; Van

Hulst, N. F.; Greve, J.; Hansma, P. K., SPIE 1992, 1639, 198.
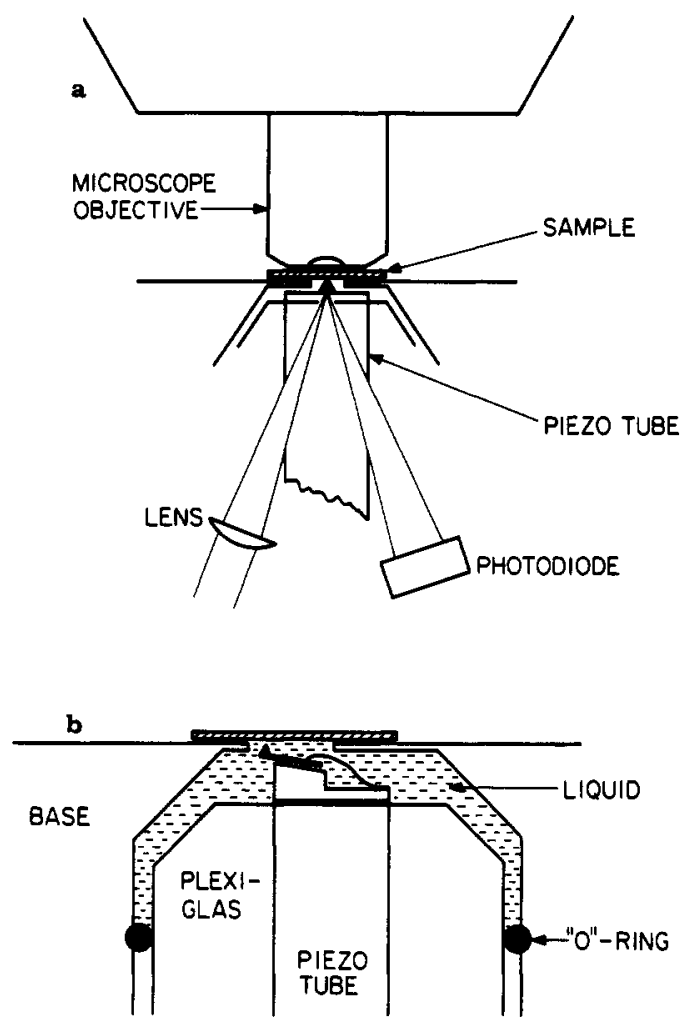

Figure 1. Schematic diagram of the combined atomic force microscope-fluorescence microscope. (a) (front view) Light from a laser diode is focused on the backside of the cantilever with integrated pyramidal tip (dark triangle). Deflections of the cantilever are detected as shifts of the laser beam at the photodiode. A feedback loop keeps the deflection constant by moving the cantilever up and down with the piezo tube. The microscope objective of the fluorescence microscope gives an image of the sample and of the cantilever. An object can be selected and moved with a translation stage (not shown here) to the AFM tip to be imaged with high resolution. (b) (side view) The AFM can be operated in air and under liquid. The liquid cell is sealed with a rubber O-ring at the bottom and by the sample at the top. A flexible O-ring between the Plexiglas cone and piezo tube (not shown here) seals the piezo tube from the liquid, but it allows the piezo tube to scan the cantilever with negligible restrictions. The cantilever is drawn larger than to scale to make it visible.

In the AFM design most widely used, ${ }^{28}$ a tripod construction is used to engage the tip by tilting the head (mechanical assembly holding laser diode, photodiode, and cantilever) toward the sample. In the design presented here, two leaf springs attached to the frame holding the AFM and a micropositioning screw (in contact with the bottom side of the base plate on top of which the sample is placed) driven by a stepper motor are used to give a linear engagement without rotation. In this way, the fluid cell stays sealed and the forces between the rubber O-ring and the Plexiglas (Figure 1b) generated by a rotational motion are minimized.

The AFM is operated by the feedback electronics and the data-acquisition system of the commercially available Nanoscope II (Digital Instruments, Santa Barbara, CA). The maximum scan range of the piezo is $6 \times 6 \mu \mathrm{m}^{2}$. In the images presented here, we used $200-\mu \mathrm{m}$-long microfabricated cantilevers with integrated pyramidal tips (Digital Instruments), having a force constant of $0.06 \mathrm{~N} / \mathrm{m}$. The applied force in air between tip and sample has a typical value of $10 \mathrm{nN}$. In water this force is lower (a few nanonewtong) due to the lack of the adhesion forces. ${ }^{20}$ The AFM images (400 $\times 400$ data points) shown here are obtained at a rate of 8.7 lines/s for low magnification images and at a rate

(28) Hansma, P. K.; Eling, V. B.; Marti, O.; Bracker, C. E. Science 1988, 242, 209.

(29) Weisenhorn, A. L.; Hansma, P. K.; Albrecht, T. R.; Quate, C. F. Appl. Phys. Lett, 1989, 54, 2651. 

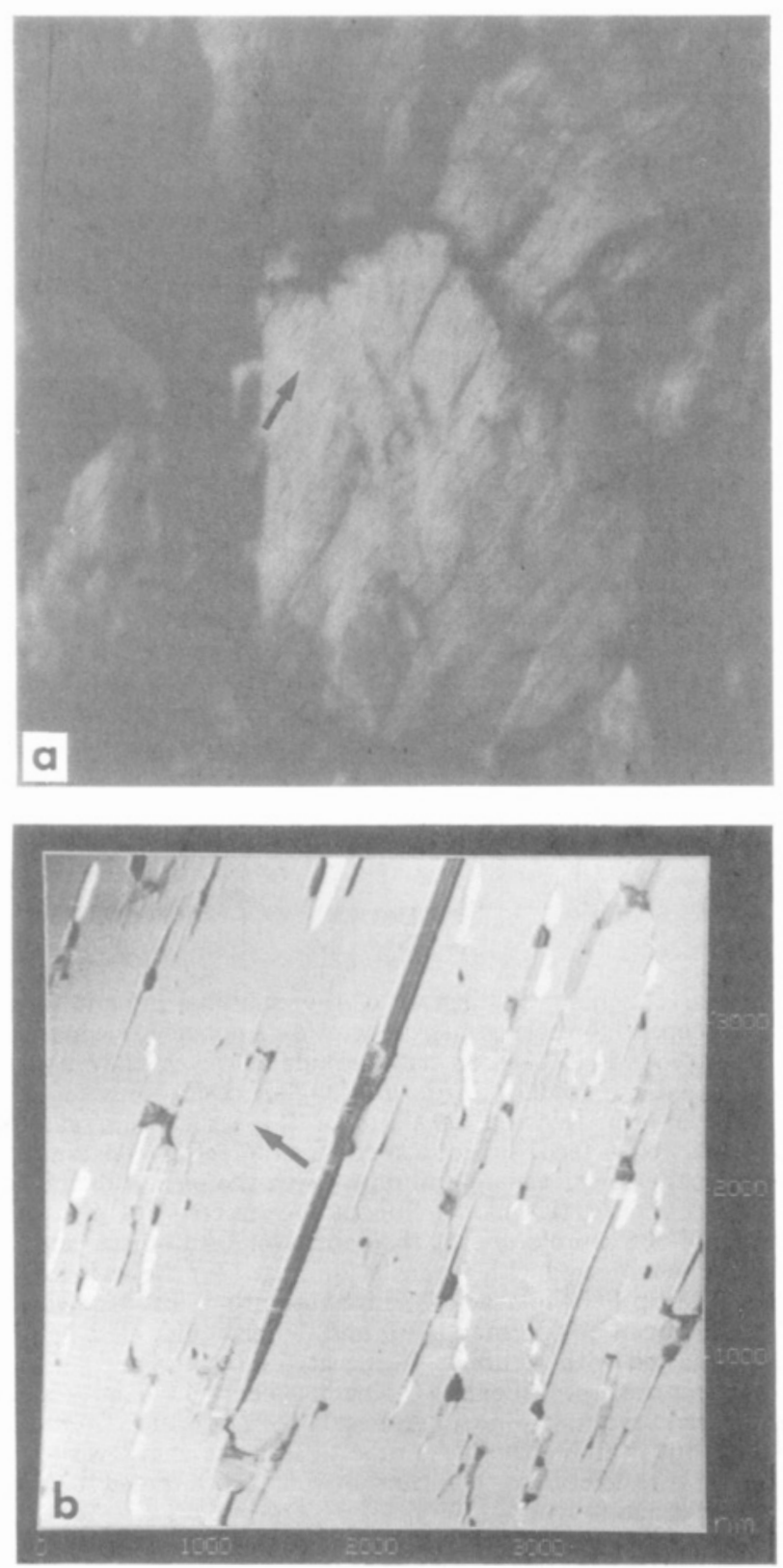
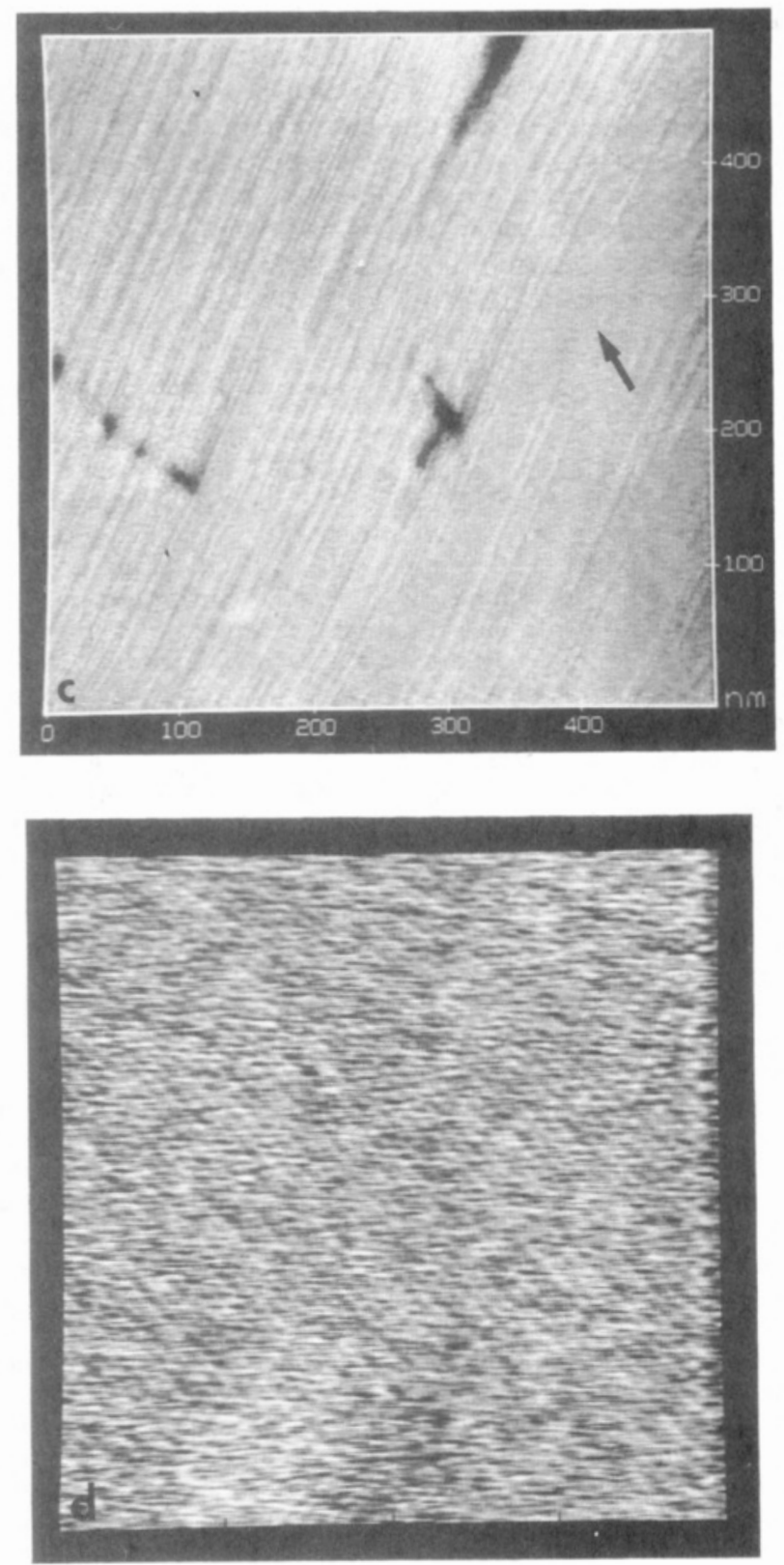

Figure 2. Polymerized LB film of PCA on muscovite mica as imaged by the fluorescence microscope (a) and by the AFM in air (b-d). (a) The polarization of the excitation light is along the direction of the cracks in the film indicated by the arrow: microscope objective, $40 \times$; excitation/barrier, $546 \mathrm{~nm} / 610 \mathrm{~nm}$; image size; $240 \times 240 \mu \mathrm{m}^{2}$. The following image sizes were used for the AFM: (b) $4 \times 4 \mu \mathrm{m}^{2}$, (c) $500 \times 500 \mathrm{~nm}^{2}$, (d) $20 \times 20 \mathrm{~nm}^{2}$. Arrows indicate regions where each subsequent image has been obtained. AFM images have been leveled but not further filtered.

of 39 lines/s for molecular resolution images. In the AFM images light regions correspond to high features opposed to dark regions which correspond to low features. Samples can be changed within $2 \mathrm{~min}$, even when imaging under liquid, enabling a high sample throughput.

The AFM is integrated with a fluorescence microscope (AXIOSKOP, Zeiss, FRG) by replacing the normal sample stage by a new stage for the AFM. The fluorescence microscope is equipped with two objectives: $10 \times, 0.25 \mathrm{NA}$, and $40 \times, 0.65 \mathrm{NA}$ (NA = numerical aperature). Since the light transverses first glass or mica, the effective NA will be lower. Assuming glass (refractive index 1.55) as the sample substrate, the NA of the $40 \times$ objective will be 0.4 . Subsequently the optical resolution is about $1 \mu \mathrm{m}$. The microscope carries two filter combinations for fluorescence imaging: excitation/barrier $=(1) 450-490 \mathrm{~nm}$ (blue)/ $520 \mathrm{~nm}$ (yellow) and (2) $546 \mathrm{~nm}$ (green) $/ 610 \mathrm{~nm}$ (red). Since the bright light transmission unit (luminous field diaphragm and condenser), the sample stage, and the sample stage carrier have been removed to create space for the AFM, a bright light reflection channel is created using a UV filter and a neutral density filter to reduce the light level coming from the $100-\mathrm{W} \mathrm{Hg}$ arc lamp. To the dovetail of the fluorescence microscope, directly connected to the coaxial coarse/fine focusing control, a new plate is connected on which the AFM is placed. When working in fluorescence, the cantilever with integral tip is not visible. The AFM tip is centered in line with the microscope objective and a cross wire in the eyepiece by moving the whole AFM with an $x y$-stage illuminated by the bright light. The cross wire is still visible (but faintly) when fluorescence is used, and thus the position of the tip is known. However, the tip can be visualized even while working in fluorescence, using the transmission image of the cantilever generated simultaneously by the light from the laser diode. The sample on top of the AFM is brought in focus by moving the AFM up and down with the focusing control knob of the fluorescence microscope and can be translated using a second stage.

\section{Results}

Cracks in the polymerized PCA LB film are revealed by the fluorescence microscope and AFM. In Figure 2a the LB film is shown as seen with the fluorescence microscope. The polarization direction of the excitation light is parallel 

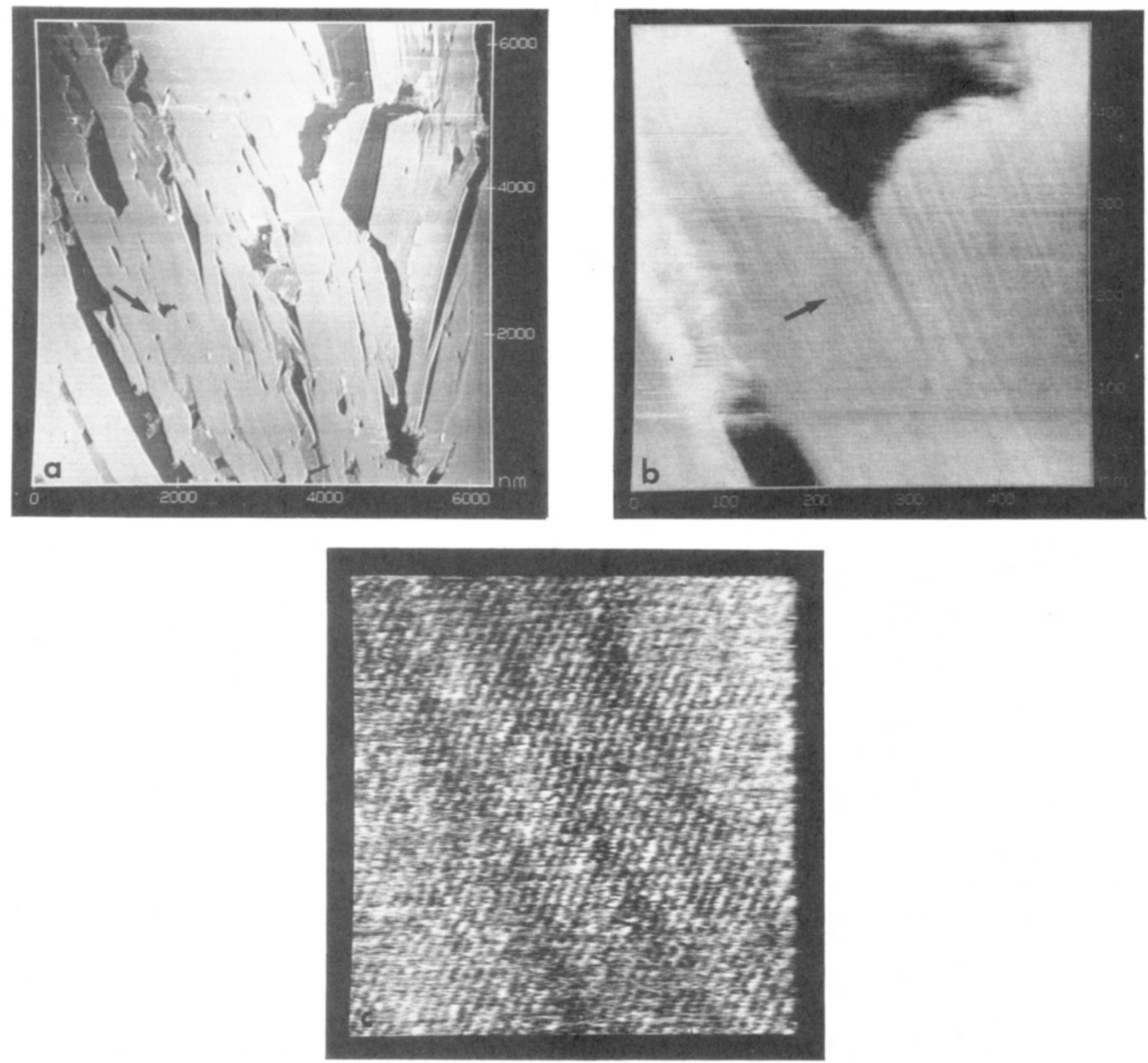

Figure 3. Polymerized LB film of PCA on muscovite mica imaged with the AFM in air. The following image sizes were used: (a) $6.3 \times 6.3 \mu \mathrm{m}^{2}$, (b) $500 \times 500 \mathrm{~nm}^{2}$, (c) $15 \times 15 \mathrm{~nm}^{2}$. Arrows indicate regions where each subsequent image has been obtained. Images have been leveled only.

along the cracks (indicated by the arrow). When the polarization direction is changed, the fluorescence diminishes to a negligible level at the polarization direction perpendicular to cracks. This is in agreement with recent observations by Goettgens et al. ${ }^{20}$ which show that the fluorescence from polymerized PCA LB films is highly polarized (polarization degree $>0.95$ ) at high surface pressures. The area indicated with the arrow in Figure 2a was imaged with the AFM in air. The directions of the cracks clearly have the same orientation (Figure 2b). Cross sections through the AFM image in Figure $2 \mathrm{~b}$ and other images reveal plateaus corresponding to one-, three-, and five-layer films. Trilayer films are most predominant, and it is on these pieces of film that molecular resolution images have been obtained. Higher magnification AFM images reveal stripe-like corrugations on the LB film that have the same orientation as the cracks (Figure 2c). These corrugations are not due to roughness of the mica substrate, but may be due to warping of the LB films during compression in the Langmuir through and subsequent transfer to mica, or they may be inherent in the polym- erization process. The molecular resolution image showing the tailgroups (Figure 2d) clearly indicates that one of the crystal lattice directions coincides with the directions of the cracks. The molecular packing is in agreement with earlier observations by X-ray and electron diffraction on multilayers. $^{8}$

It has been suggested that the cracks are parallel to one of the crystal axes (including the direction of the polymer backbone) of the LB film. ${ }^{15}$ It is known that the fluorescence from the LB film is polarized along the direction of the polymer backbone. ${ }^{20,30}$ From several other series of fluorescent images compared to AFM images of the same region, it was observed that the fluorescent light is highly polarized and has its direction parallel to the cracks in the LB films. Thus, the film breaks parallel to the polymer backbone which is confirmed by the lattice directions revealed in the molecular resolution image (Figure 2d). The high degree of polarization also indicates

(30) Göbel, H. D.; Gaub, H. E.; Möhwald, H., Phys. Lett. 1987, 138, 

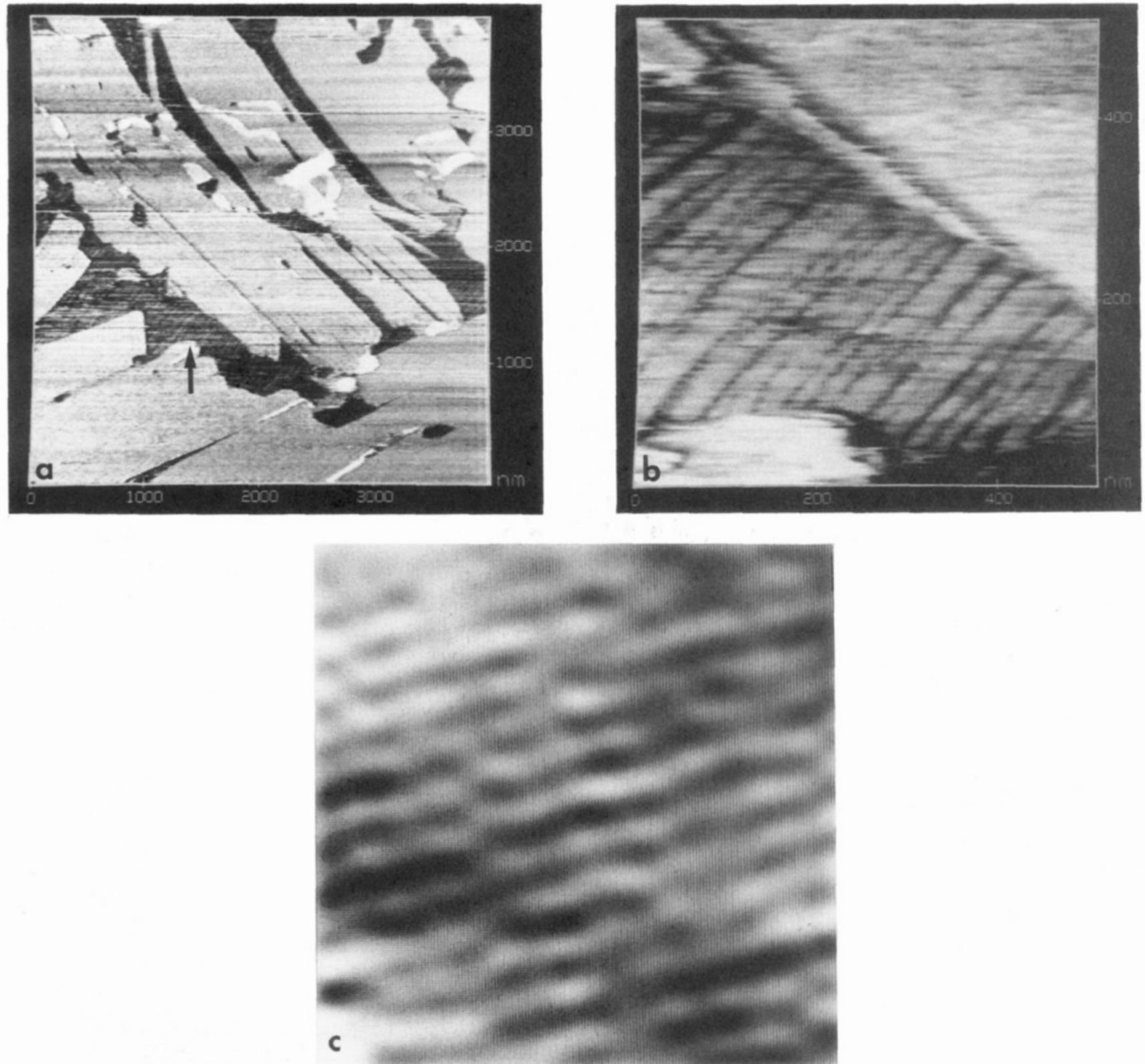

Figure 4. Polymerized LB film of PCA on Cd-PCA imaged with the AFM under water. The area indicated by the arrow in (a) is imaged in (b). (c) Shows the image of the upper (flat) surface of the top LB film. The following image sizes were used: (a) $4 \times 4$ $\mu \mathrm{m}^{2}$, (b) $500 \times 500 \mathrm{~nm}^{2}$, (c) $5 \times 5 \mathrm{~nm}^{2}$. All images have been leveled, and in addition (c) has been Fourier filtered to remove artifacts related to the scanning.

that the multiple layers in the LB film have their polymer backbone arranged parallel to each other.

Another series of images take in air (Figure 3) shows again the ridges due to warping of the LB film during compression. As observed in Figure 2 the ridges are oriented in the direction of the cracks. In Figure $3 \mathrm{c}$ it is evident that the direction of the ridges coincides with one of the lattice directions, the direction of the polymer backbone. This appearance of ridges at the various levels of magnifications has been previously observed on UV light polymerized LB films of BRONCO. ${ }^{16}$ Possible explanations for this feature are the following. Firstly, cracks in the bottom layer are smoothly covered by the top layer, which is slightly indented over the cracks. Secondly, the formation of these ridges is inherent in the polymerization process. During the UV irradiation stretches of polymer will be formed randomly, not simultaneously. This can cause mechanical stress, resulting in defects, such as an offset of adjacent polymers. In fact, if one follows the array of molecules going across the valleys (Figure $3 \mathrm{c}$ ), a slight zigzag movement can be observed. This zigzag pattern might indicate an offset between adjacent polymer chains.

The second sample, PCA on Cd-PCA, has been imaged under water. Figure 4a shows patches of PCA film on a layer of Cd-PCA. Zooming in between the cracks in the PCA film reveals the underlying Cd-PCA layer (Figure 4b). The orientation of this layer does not appear to be fixed with respect to the orientation of the PCA layer on top. The Cd-PCA film clearly has a different structure than the PCA film. It consists of 20-50-nm-wide filaments arranged parallel to each other. This has not been observed on Cd-PCA films imaged in air (data not shown here). Contact with water may cause the breaking up of the film into the filaments, which is energetically more favorable. The hydrophobic monolayer breaks up along the direction of its polymer backbone, collapsing onto itself to form a bilayer, exposing the hydrophilic headgroups to water. A molecular resolution image of the upper PCA layer revealing the headgroups is shown in Figure 4c. 


\section{Discussion}

An AFM integrated with a fluorescence microscope has revealed subtle details of a polymerized LB film. Whereas other techniques, such as X-ray and electron diffraction, are useful for obtaining quantative data on the lattice parameters, ${ }^{8}$ the AFM is especially suitable for investigating the local structure of LB films on a submicrometer scale. Especially on biological samples, this combination of microscopes may become a new valuable tool for highresolution imaging; it combines object location and selection with the optical microscope and high-resolution imaging with the AFM. Samples ranging from chromosomes, cells, and cell organelles to DNA are nowadays routinely labeled with a growing number of fluorescent labels. Now additional structural information on these samples can be gathered with a combined AFM-fluorescence microscope.

This design for a combined AFM-fluorescence microscope uses a standard rather than an inverted microscope. Thus, the sample serves as a lid on the fluid cell, allowing one to change samples readily without emptying the fluid cell. Furthermore, the optical beam deflection system (laser diode, cantilever, photodiode) stays intact, enabling a high throughput of samples.

As a demonstration of the power of the combined AFMfluorescence microscope, we have shown here a direct correlation between the cracks seen in the polymerized PCA LB film with the fluorescence microscope and the molecular arrangement in the crystal as revealed by the AFM. These results confirm that the LB film breaks along the direction of the polymer backbone.

Acknowledgment. We thank Matt Wilson for his help in the design of the microscope and for machining the mechanical parts. We thank Srinivas Manne for technical assistance. This research was supported by Digital Instruments (6780 Cortona Dr., Santa Barbara, CA 93117), the Netherlands Organization for Scientific Research NWO (C.A.J.P.), and the National Science Foundation (H.G.H., P.K.H.).

Registry No. PCA (homopolymer), 66990-33-8. 